\title{
Correction to: Primary immunodeficiencies in Central and Eastern Europe-the power of networking Report on the activity of the Jeffrey Modell Foundation Centers Network in Central and Eastern Europe
}

\author{
A. Sediva ${ }^{1}$ - M. Bataneant ${ }^{2} \cdot$ M. Belevtsev ${ }^{3} \cdot$ A. Blaziene ${ }^{4} \cdot$ P. Ciznar ${ }^{5} \cdot$ E. Förster-Waldl ${ }^{6} \cdot$ J. Kelecic $^{7} \cdot$ J. Marodi $^{8}$. \\ E. Naumova ${ }^{9}$ - G. Nasrullayeva ${ }^{10} \cdot$ K. Ress ${ }^{11} \cdot$ M. Serban ${ }^{12} \cdot$ B. Sitkauskiene ${ }^{13} \cdot$ B. Toth ${ }^{14} \cdot$ V. Modell $^{15} \cdot$ F. Modell $^{15}$. $^{15}$ \\ V. Tenembaum ${ }^{15} \cdot$ M. Marković ${ }^{16} \cdot$ T. Avcin $^{17}$
}

(C) Springer Science+Business Media, LLC, part of Springer Nature 2020

Correction to: Immunologic Research (2019) 67:358-367 https://doi.org/10.1007/s12026-019-09093-9

Due to authors' internal mistake they have misspelled the surname of one of the co-authors in this article. The surname of author number 13, was spelled as "Sitkaustiene", but it should be Sitkauskiene.

The online version of the original article can be found at https://doi.org/10. 1007/s12026-019-09093-9

A. Sediva

anna.sediva@fnmotol.cz

1 Department of Immunology, 2nd Faculty of Medicine Charles University, Motol University Hospital, Prague, Czech Republic

2 University of Medicine and Pharmacy "Victor Babes", Timisoara, Romania

3 Research Department, Belarusian Center for Pediatric Oncology, Hematology and Immunology, Minsk, Belarus

4 Center of Primary Immunodeficiencies, Vilnius University Hospital Santaros Klinikos, Vilnius, Lithuania

5 Pediatric Department, Children University Hospital in Bratislava, Comenius University in Bratislava, Faculty of Medicine, Bratislava, Slovakia

6 Department of Pediatrics \& Adolescent Medicine, Division of Neonatology, Ped. Intensive Care and Neuropediatrics, Center for Congenital Immunodeficiencies, Vienna, Austria

7 Department of Pediatrics, Division of Clinical Immunology, Allergology, Respiratory Diseases and Rheumatology, University Hospital Center Zagreb, Zagreb, Croatia
Publisher's note Springer Nature remains neutral with regard to jurisdictional claims in published maps and institutional affiliations.

8 Primary Immunodeficiency Clinical Unit \& Laboratory, Department of Dermatology, Venerology \& Dermatooncology, Semmelweis University, Budapest, Hungary

9 Clinical Immunology \& Stem Cell Bank, University Hospital "Aleksandrovska", Sofia, Bulgaria

10 Department of Allergy and Immunology, Azerbaijan Medical University, Baku, Azerbaijan

11 Center of Allergology and Immunology, East Tallinn Central Hospital, Tallinn, Estonia

12 Clinical Emergency Hospital for Children Louis Turcanu, Timisoara, Romania

13 Department of Immunology and Allergology, Center of Primary Immunodeficiency, Lithuanian University of Health Sciences, Kaunas, Lithuania

14 Department of Infectious and Pediatric Immunology, Faculty of Medicine, University of Debrecen, Debrecen, Hungary

15 The Jeffrey Modell Foundation, New York, NY, USA

16 Octapharma Nordic AB, Stockholm, Sweden

17 Department of Allergology, Rheumatology and Clinical Immunology, University Children's Hospital, University Medical Center Ljubljana, Ljubljana, Slovenia 